\title{
EFEITO DA CARGA DE SÓLIDOS E DE ENZIMAS NA HIDRÓLISE DA PALHA DE CANA-DE-AÇÚCAR
}

\author{
R. B. SOUZA ${ }^{1}$, B. PRATTO ${ }^{1}$, J. D. ANGARITA ${ }^{2}$, A. R. SECCHI $^{2}$ e A. J. G. CRUZ ${ }^{1}$ \\ ${ }^{1}$ Universidade Federal de São Carlos, PPGEQ-UFSCar, Programa de Pós-Graduação em \\ Engenharia Química \\ ${ }^{2}$ Universidade Federal de Rio de Janeiro, COPPE, Programa de Engenharia Química \\ E-mail para contato: renata.beraldo@yahoo.com.br
}

\begin{abstract}
RESUMO - A hidrólise enzimática é a uma das principais etapas na conversão da biomassa em etanol. Este trabalho estudou o efeito da carga de sólidos e de enzimas na hidrólise enzimática da palha da cana-de-açúcar pré-tratada hidrotermicamente $\left(195{ }^{\circ} \mathrm{C}\right.$, 10 min, razão sól.-líq. 1:10). Os experimentos foram realizados em frascos de Erlenmeyer a $50^{\circ} \mathrm{C}, 250 \mathrm{rpm}$ e $\mathrm{pH} \mathrm{4,8}$ (volume reacional de $50 \mathrm{~mL}$ ). Dois conjuntos de experimentos foram realizados. No primeiro avaliou-se o efeito da carga de sólidos $(10,15$ e $20 \% \mathrm{~m} / \mathrm{v})$ mantendo a carga enzimática em $10 \mathrm{FPU} / \mathrm{g}_{\text {celulose }}$. No segundo conjunto avaliou-se o efeito da carga de enzimas $\left(5,10,15,20\right.$ e $\left.30 \mathrm{FPU} / \mathrm{g}_{\text {celulose }}\right)$ mantendo a carga de sólidos em 15\%. Em 72h de hidrólise as conversões de celulose obtidas foram: 51,6\% (10\% de sólidos), 50,9\% (15\% de sólidos) e 48,2\% (20\% de sólidos). Nos experimentos realizados com $15 \%$ de sólidos as conversões obtidas foram: 41,7\% (5 FPU/g $\left.\mathrm{g}_{\text {celulose }}\right), 50,9 \%$ (10 FPU/g $\left./ \mathrm{g}_{\text {celulose }}\right), \quad 58,8 \%$ (15 FPU/g $\left./ g_{\text {celulose }}\right), 62,5 \%$ (20 FPU/g $\left./ g_{\text {celulose }}\right)$ e 63,2\% (30 FPU/g $\left.g_{\text {celulose }}\right)$. Considerou-se como melhor resultado o ensaio com $15 \%$ de sólidos e $20 \mathrm{FPU} / \mathrm{g}_{\text {celulose }}$.
\end{abstract}

\section{INTRODUÇÃO}

Nos últimos anos com a crescente preocupação com a preservação ambiental juntamente com os problemas de oferta e preço de petróleo, tem-se intensificado a busca por novos combustíveis que sejam renováveis e sustentáveis. Nesse contexto o etanol derivado de biomassas lignocelulósicas residuais surge como uma alternativa promissora, pois a utilização desses materiais além de apresentar facilidade pela sua disponibilidade e baixo custo, não acarretam em um aumento da área cultivada nem ocasionam aumento de preço ou diminuição da disponibilidade de alimentos.

O processamento da cana-de-açúcar para a produção de açúcar e etanol gera uma grande quantidade de resíduos agrícolas dentre eles a palha. Grande parte desta biomassa é deixada no campo e poderia ser utilizada para aumentar a produção de bioetanol. Para cada tonelada de cana processada são gerados cerca de $280 \mathrm{~kg}$ de resíduos, divididos entre aproximadamente $140 \mathrm{~kg}$ de palha e $140 \mathrm{~kg}$ de bagaço em base seca (Santos et al., 2012). Teoricamente cada tonelada de palha (base seca) poderia gerar cerca de 280 litros de etanol a partir da celulose. 


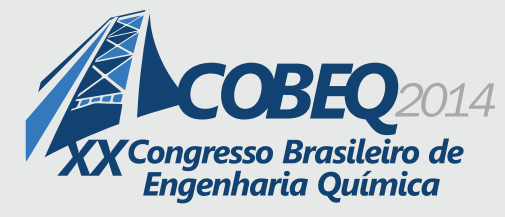

Os materiais lignocelulósicos são complexos orgânicos de carbono cuja estrutura é principalmente representada pela interação físico-química entre a celulose (polímero linear formado por unidades de $\beta$-(1,4)-D-glucopiranose unidas entre si por ligações inter e intra-moleculares de hidrogênio), a hemicelulose (heteropolímero altamente ramificado formado principalmente por açúcares contendo cinco átomos de carbono) e a lignina (macromolécula aromática de alta massa molar) (Ferreira-Leitão et al., 2010; Santos e Gouveia, 2009). A produção de etanol a partir de materiais lignocelulósicos envolve quatro etapas principais: pré-tratamento da biomassa (para prover a diminuição da cristalinidade da celulose e separação das três frações), hidrólise (para obtenção dos açúcares fermentescíveis), fermentação (para conversão dos açúcares em etanol) e destilação (para concentração do etanol) (Canilha et al., 2012; Santos et al., 2013).

A conversão de materiais lignocelulósicos em açúcares fermentecíveis pode ser feita tanto por via ácida como por via enzimática. O uso de enzimas apresenta várias vantagens quando comparada à rota ácida. Citam-se menores custos de equipamento (pelo fato da reação ser realizada em condições mais amenas) e menor formação de subprodutos que podem trazer prejuízos na etapa de fermentação (Cara et al., 2007). No entanto, o uso de enzimas ainda apresenta alto custo. Assim, o estudo da carga de sólidos e da dosagem de enzimas empregada é fator de grande importância.

Muitos fatores têm contribuído para o elevado custo da hidrólise enzimática, como os efeitos de inibição das enzimas, a acessibilidade das enzimas ao substrato e a ligação não produtiva, exigindo o uso de altas concentrações de enzimas e tornando a carga enzimática uma importante variável do processo (Leu e Zhu, 2012; Mussato et al., 2008).

Outra variável importante na hidrólise enzimática é a carga de sólidos. O aumento de carga de sólidos é fundamental para a produção industrial, assim é possível alcançar elevadas concentrações de açúcares o que traz economia significativa no processo como diminuição do custo operacional das etapas de hidrólise e fermentação e minimização do consumo de energia nas etapas de evaporação/destilação reduzindo assim o custo de produção (Ioelovich e Morag, 2012).

O aumento da carga de substrato, a redução da carga enzimática e do tempo de residência associados às melhorias na atividade do complexo enzimático são fatores que contribuem para a viabilidade da rota enzimática (Ouyang et al., 2013). Para o processo de hidrólise enzimática ser realizado com elevada eficiência, é necessária a otimização das condições de hidrólise. Desta forma este trabalho propôs avaliar o efeito da carga de sólidos e de enzimas na hidrólise enzimática da palha da cana-de-açúcar pré-tratada hidrotermicamente.

\section{MATERIAIS E MÉTODOS}

\subsection{Materiais}

A palha de cana-de-açúcar in natura utilizada neste trabalho foi gentilmente cedida pela Usina Itapira (Catanduva, SP). O complexo enzimático Cellic ${ }^{\circledR} \mathrm{CTec} 2$, composto por celulases, $\beta$-glicosidases e hemicelulases tem uma atividade medida em termos de unidade de papel de filtro de 


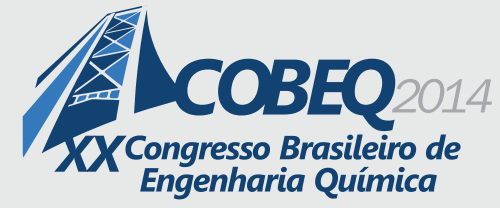

203 FPU/ml (Ghose, 1987). Este complexo foi doado pela Novozymes Latin America (Araucária, PR).

\subsection{Métodos}

Pré-tratamento hidrotérmico: O pré-tratamento hidrotérmico foi realizado em reator de aço inoxidável (modelo 4532, Parr Instruments Company, Moline, Illinois, EUA). A palha de cana-deaçúcar in-natura previamente seca e moída foi submetida à temperatura de $195^{\circ} \mathrm{C}$ por 10 min na proporção $1: 10 \mathrm{~m} / \mathrm{v}(200 \mathrm{rpm})$. Ao final da reação a fração sólida foi separada por filtração e lavada com água para remoção dos componentes solubilizados. Parte da fração sólida foi reservada para a determinação da umidade e caracterização química.

Caracterização das amostras: As amostras foram caracterizadas de acordo com procedimento descrito por Gouveia et al. (2009).

Hidrólise enzimática: $\mathrm{O}$ material pré-tratado foi submetido à hidrólise enzimática em meio contendo tampão citrato $(50 \mathrm{mM}, \mathrm{pH} 4,8)$ em um volume total de reação de $50 \mathrm{ml}$. A palha pré-tratada e o tampão foram adicionados a frascos Erlenmeyers de $250 \mathrm{~mL}$ e transferidos para o shaker $\left(50^{\circ} \mathrm{C}\right.$ e $250 \mathrm{rpm}$ ) onde permaneceram por $30 \mathrm{~min}$ para atingir o equilíbrio térmico. Após esse período, as reações de hidrólise foram iniciadas pela adição de enzimas. $\mathrm{O}$ efeito da carga de sólidos foi avaliado em experimentos contendo 10,15 e $20 \% \mathrm{~m} / \mathrm{v}$ de biomassa com $10 \mathrm{FPU} / \mathrm{g}_{\text {celulose }}$. O efeito da carga de enzimas foi avaliado mantendo-se a carga de sólidos em $15 \%$ e variando-se a carga de enzimas em 5, 10, 15,20 e $30 \mathrm{FPU} / \mathrm{g}_{\text {celulose. }}$

As concentrações de açúcares foram determinadas por análises em cromatógrafo equipado com detector de índice de refração. As amostras foram diluídas e filtradas $(0,45 \mu \mathrm{m})$ antes de serem injetadas no equipamento. Para a separação dos componentes foi utilizada a coluna Biorad Aminex $\mathrm{HPX}-87 \mathrm{H}$ na temperatura de $65^{\circ} \mathrm{C}$, fase móvel $\mathrm{H}_{2} \mathrm{SO}_{4} 5,0 \mathrm{mM}$ na vazão $0,6 \mathrm{~mL} / \mathrm{min}$.

\section{RESULTADOS E DISCUSSÕES}

Os dados da caracterização das amostras de palha (in natura e pré-tratada hidrotermicamente) e a perda/remoção dos componentes das amostras de palha submetidas às diferentes condições de prétratamento encontram-se na Tabela 1 . O fator de severidade é uma medida da intensidade das condições operacionais (tempo e temperatura) empregadas durante o pré-tratamento. Esta estimativa foi proposta por Overend et al. (1987).

Após o pré-tratamento $85,3 \%$ da hemicelulose e $32,5 \%$ da lignina foram removidos. Uma boa remoção da hemicelulose pode contribuir para a ação das enzimas celulolíticas uma vez que essa estrutura forma uma barreira física ao redor da celulose dificultando o acesso das enzimas (Diaz et al., 2010). 
Tabela 1 - Caracterização da palha antes e após pré-tratamento hidrotérmico e perda/solubilização dos componentes das amostras de palha.

\begin{tabular}{cccc}
\hline \hline Componente & $\begin{array}{c}\text { In natura } \\
\left(\mathbf{g} / \mathbf{1 0 0} \mathbf{g}_{\text {palha }}\right)\end{array}$ & $\begin{array}{c}\text { Pré-Tratamento-195 } \mathbf{C} / \mathbf{1 0} \text { min } \\
\left(\mathbf{g} / \mathbf{1 0 0} \mathbf{~ g}_{\text {palha }}\right)\end{array}$ & Perda/remoção (\%) \\
\hline Celulose & 36,7 & 31,9 & 13,1 \\
Hemicelulose & 29,9 & 4,40 & 85,3 \\
Lignina & 19,7 & 13,3 & 32,5 \\
Cinzas & 6,2 & 2,6 & 58,1 \\
Extrativos & 6,2 & - & \\
Fator de severidade $^{* *}(-)$ & - & 3,60 & \\
\hline \hline
\end{tabular}

${ }^{*}$ Rendimento mássico $=\left(\mathrm{m}_{\text {final amostra após pré-tratamento (BS) }} / \mathrm{m}_{\text {inicial amostra (BS) }}\right) \times 100=51,8 \%$

** Estimando segundo Overend et al. (1987)

Os perfis de concentração de glicose e conversão em função do tempo de hidrólise para as três cargas de sólidos avaliadas são apresentados na Figura 1(a) e (b) respectivamente.
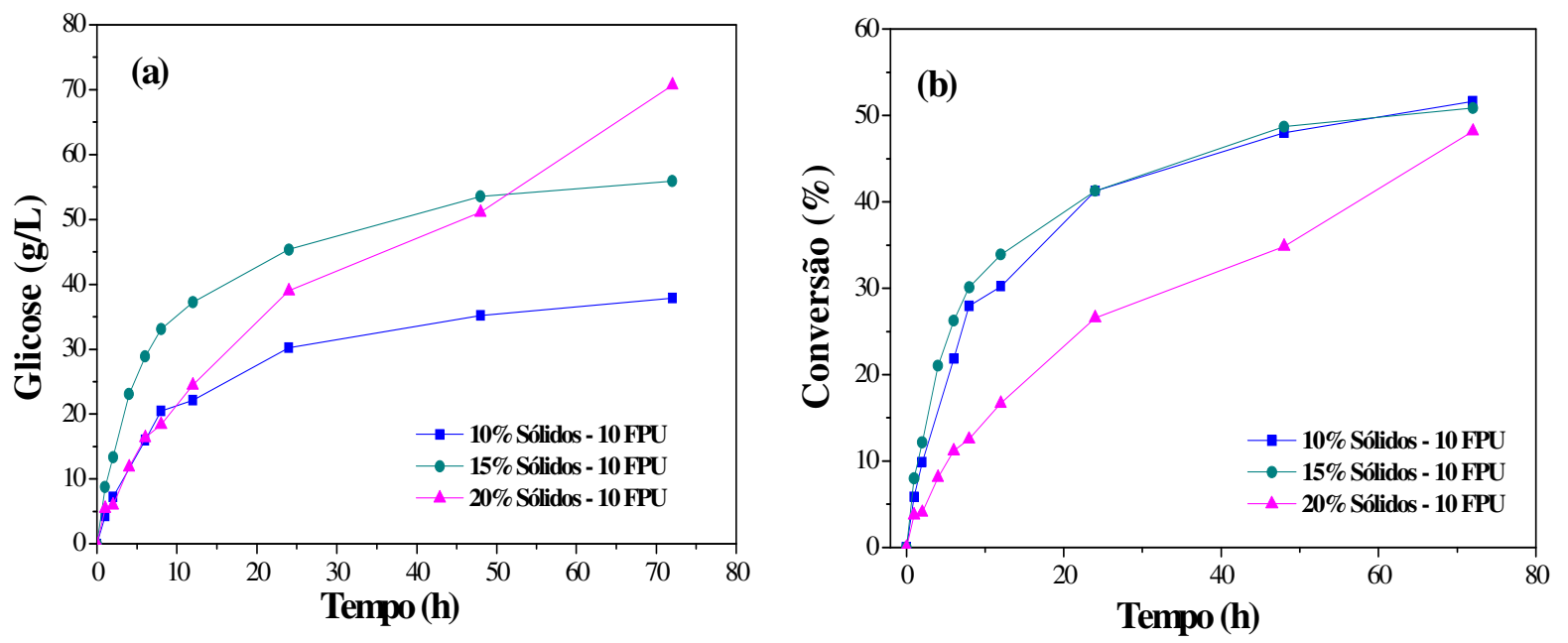

Figura 1 - Perfil de concentração de glicose em g/L (a) e conversão da celulose (b) em função do tempo de hidrólise para cargas de sólidos de 10, 15 e 20\% de sólidos e carga enzimática de 10 FPU/g celulose.

O ensaio realizado com $10 \%$ de sólidos resultou em uma concentração de glicose de 37,9 g/L (em 72 h), com conversão de celulose de 51,6\%. Quando a carga de sólidos foi aumentada para 20\%, a concentração de glicose atingiu 70,7 g/L (em $72 \mathrm{~h}$ ), cerca de $86,7 \%$ maior que a obtida com $10 \%$ de sólidos, porém a conversão nesta condição foi de $48,2 \%$. Para a carga de sólidos de $15 \%$ obteve-se $55,9 \mathrm{~g} / \mathrm{L}$ de glicose (em $72 \mathrm{~h}$ ), conversão de 50,9\%. Pode-se observar pelos resultados que o aumento na carga de sólidos ocasiona um aumento na concentração final de glicose demonstrando um comportamento aproximadamente linear entra a carga de sólidos e a concentração de glicose final, 


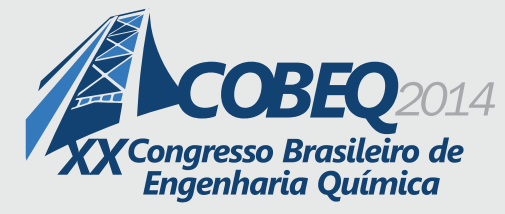

como observado por López-Linares et al. (2014) com palha de colza. Segundo Ramachandriya et al. (2013), é importante operar em concentrações altas de sólidos a fim de se obter altas concentrações de etanol na fermentação, reduzindo assim o consumo de energia na destilação.

A partir dos resultados obtidos decidiu-se avaliar o efeito da carga de enzimas em experimentos empregando 15\% de carga de sólidos, em função da maior conversão de celulose obtida.

Na Figura 2 são mostrados respectivamente os resultados de concentração de glicose e conversão durante a hidrólise com diferentes cargas enzimáticas. Os resultados obtidos demonstram que o aumento da carga de enzimas promoveu uma maior liberação de glicose e melhor eficiência na conversão da celulose, no entanto cargas enzimáticas acima de $20 \mathrm{FPU} / \mathrm{g}_{\text {celulose }}$ não apresentaram uma melhora na conversão e na concentração de glicose liberada. Resultados semelhantes foram obtidos por Chen et al. (2007) utilizando como substrato sabugo de milho, Caspeta et al. (2014) com bagaço de agave e por Mesa et al. (2010) que durante a hidrólise do bagaço de cana a variação das cargas de enzimas de 15 e $25 \mathrm{FPU} / \mathrm{g}_{\text {celulose }}$ não apresentaram diferença significativa na concentração de glicose liberada no final do ensaio.
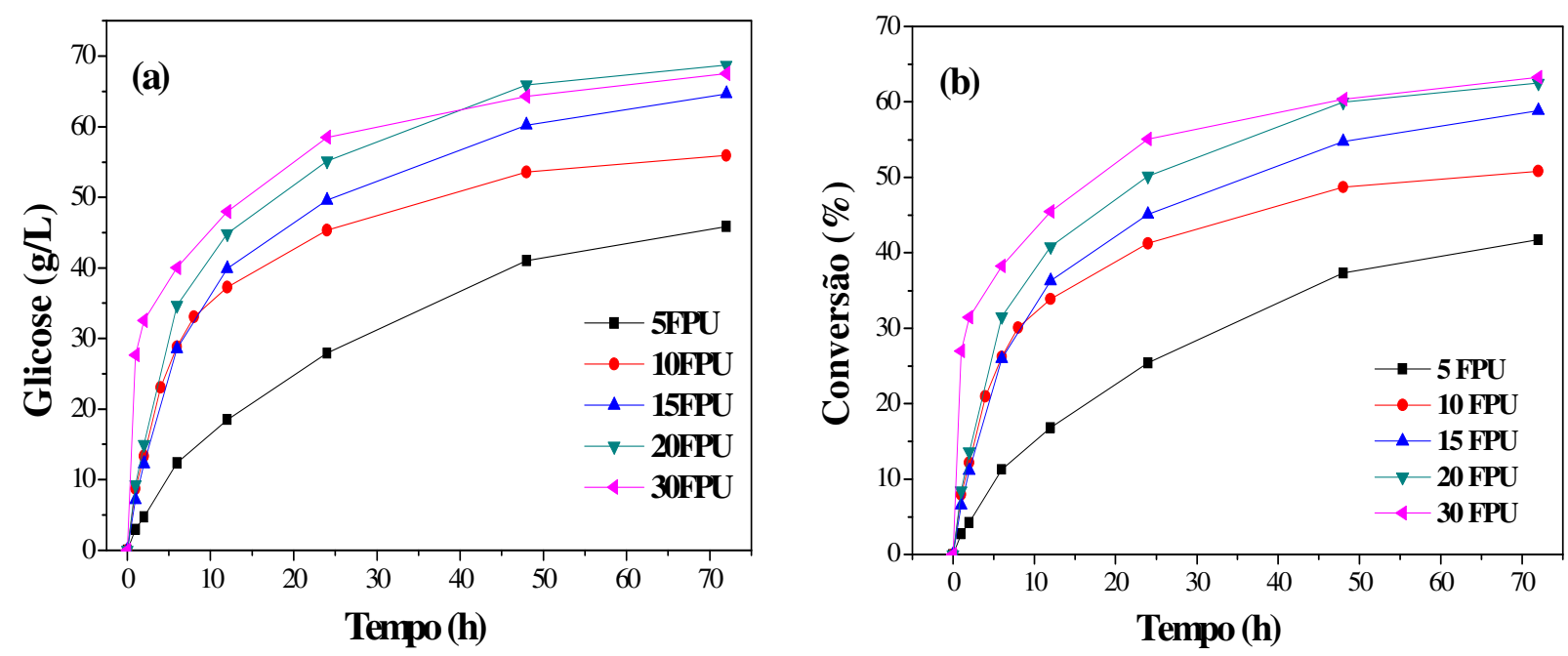

Figura 2 - Perfil de concentração de glicose em g/L (a) e conversão da celulose (b) em função

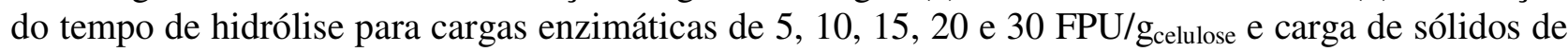
$15 \%$.

O aumento da carga de enzimas de 20 para $30 \mathrm{FPU} / \mathrm{g}_{\text {celulose }}$ apresentou um aumento na velocidade inicial da hidrólise. Contudo, esse fator não compensa o uso dessa carga visto que após $72 \mathrm{~h}$ as conversões obtidas nos dois experimentos foram muito próximas $(62,5 \%$ e 63,2\% de conversão para os experimentos com 20 e $30 \mathrm{FPU} / \mathrm{g}_{\text {celulose }}$ respectivamente). $\mathrm{O}$ custo do complexo enzimático contribui significativamente para o custo total do processo de conversão da biomassa, desta forma a dosagem da enzima deve ser minimizada (Chen et al., 2007). 
A Figura 3 apresenta a conversão de celulose em função da carga de enzimas em 72h. Observase o aumento da conversão com o aumento da carga de enzimas até $20 \mathrm{FPU} / \mathrm{g}_{\text {celulose }}$. Uma possível explicação para esse efeito da redução da taxa de aumento da conversão com o aumento da carga de enzimas é a inibição das enzimas pela elevada concentração de glicose (cerca de $68 \mathrm{~g} / \mathrm{L}$ ), segundo Mussato et al. (2008).

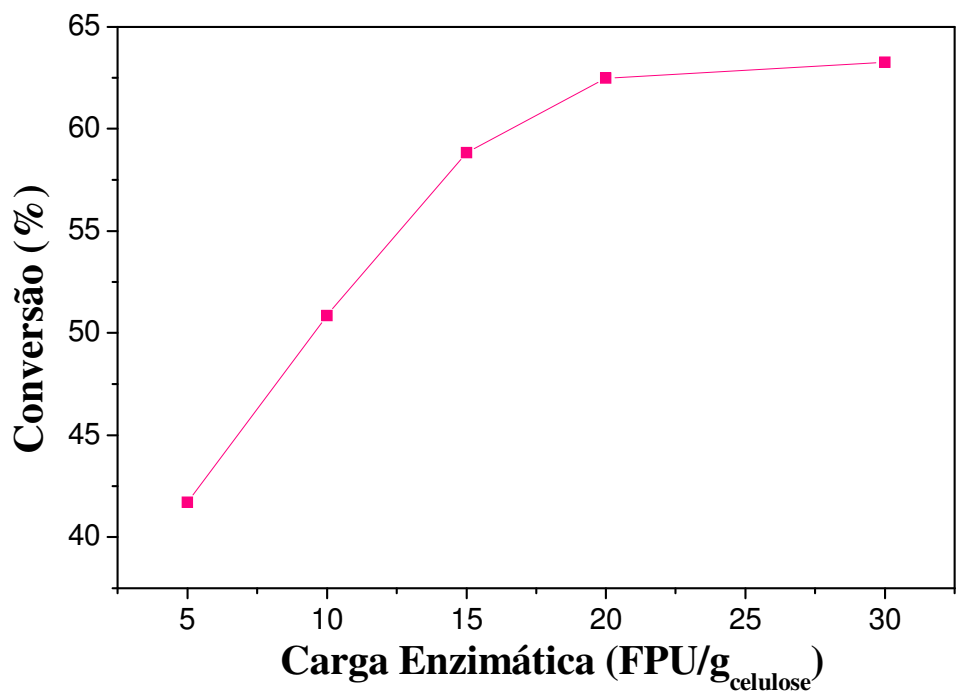

Figura 3 - Conversão da celulose em função da carga de enzimas emprega na hidrólise (em 72 h).

\section{CONCLUSÃO}

Com relação à carga de enzimas o aumento desta variável promove uma maior liberação de glicose e eficiência de conversão. No entanto para valores acima de $20 \mathrm{FPU} / \mathrm{g}_{\text {celulose }}$ não houve ganhos consideráveis na conversão. A fim de se obter altas concentrações de glicose ao final da etapa de hidrólise, para que se obtenham concentrações mais elevadas de etanol na etapa de fermentação, deve-se encontrar uma boa relação entre a carga de sólidos e de enzimas a ser utilizada. Desta forma, a partir dos resultados obtidos neste trabalho, concluiu-se que a melhor condição experimental foi

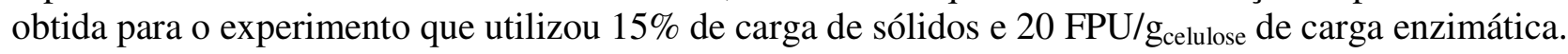

\section{AGRADECIMENTOS}

Os autores agradecem à CAPES, ao CNPq e ao Programa de Recursos Humanos da Agência Nacional de Petróleo, Gás e Biocombustíveis (PRH 44-ANP), pelo apoio financeiro para realização deste trabalho. 


\section{REFERÊNCIAS BIBLIOGRÁFICAS}

CANILHA, L.; CHANDEL, A. K.; MILESSI, T. S. S.; ANTUNES, F. A. F.; FREITAS, W. L. C.; FELIPE, M. G. A.; SILVA, S. S. Bioconversion of sugarcane biomas sinto ethanol: an overview about composition, pretreatment methods, detoxification of hydrolysates, enzymatic saccharification, and ethanol fermentation. J Biomed Biotechnol, v. 2012, 15p, 2012.

CARA, C.; MOYA, M.; BALLESTEROS, I.; NEGRO, M. J.;GONZÁLEZ, A.; RUIZ, E. Influence of solid loading on enzymatic hydrolysis of steam explode dor liquid hot water pretreated olive tree biomass. Process Biochemistry, v. 42, p. 1003-1009, 2007.

CASPETA, L.; CARO-BERMÚDEZ, M. A.; PONCE-NOYOLA, T.; MARTINEZ, A. Enzymatic hydrolysis at high-solids loadings for the conversion of agave bagasse to fuel ethanol. Appl Energ, v. 113, p. 277-286, 2014.

CHEN, M.; XIA, L.; XUE, P. Enzymatic hydrolysis of corncob and ethanol production from cellulosic hydrolysate. Int Biodeter biodegr, v. 59, p. 85-89, 2007

DÍAZ, M. J.; CARA, C.; RUIZ, E.; ROMERO, I.; MOYA, M.; CASTRO, E. Hydrotermal prétreatment of rapeseed straw. Bioresour. Technol., v. 101, p. 2428-2435, 2010.

FERREIRA-LEITÃO, V.; GOTTSCHALK, L. M. F.; FERRARA, M. A.; NEPOMUCENO, A. L.; MOLINARI, H. B. C.; BOM, E. P. S. Biomass Residues in Brazil: Availability and Potential Uses. Waste Biomass Valor, v. 1, p. 65-76, 2010.

GHOSE, T. K. Measurement of cellulase activities. Pure \& Applied Chemistry, v. 59, p. 257-268, 1987.

GOUVEIA, E. R.; NASCIMENTO, R. T.; ROCHA, G. J. M. Validação de metodologia para a caracterização química de bagaço de cana-de-açúcar. Quím. Nova, v.32, p.1500-1503, 2009.

IOELOVICH, M.; MORAG, E. High-solids hydrolysis. BioResources, v. 7(4), p. 4672-4682, 2012.

LEU, S.; ZHU, J. Y. Substrate-related factors affecting enzymatic saccharification of lignocelluloses: Our recent understanding. Bioenerg. Res., v. 6(2), p. 405-415, 2013.

LÓPEZ-LINARES, J. C.; ROMERO, I.; CARA, C.; RUIZ, E.; MOYA, M.; CASTRO, E. Bioethanol production from rapeseed straw at high solids loading with different process configurations. Fuel, v. 122, p. 112-118, 2014.

MESA, L.; GONZÁLEZ, E.; CARA, C.; RUIZ, E.; CASTRO, E.; MUSSATTO, S. I. An approach to optimization of enzymatic hydrolysis from sugarcane bagasse based on organosolv pretreatment. J Chem Technol Biot, v. 85, p. 1092-1098, 2010.

MUSSATTO, S. I.; DRAGONE, G.; FERnANDES, M.; MILAGRES, A. M. F.; ROBERTO, I. C. The effect of agitation speed, enzyme loading and substrate concentration on enzymatic hydrolysis of cellulose from brewer's spent grain. Cellulose, v. 15, p. 711-721, 2008.

OUYANG, J.; BAOTIAN, L.; ZHANG, M.; ZHENG, Z., YU, HENG. Enzymatic hydrolysis, adsorption, and recycling during hydrolysis of bagasse sulfite pulp. Bioresour. Technol., v. 146, p. 288-293, 2013. 
OVEREND, R.P.; CHORNET, E.; GASCOIGNE, J. A. Fractionation of lignocellulosics by steamaqueous pretreatments. Philos. Trans. R. Soc. Lond., A, v. 321(1561): 523-536, 1987.

RAMACHANDRIYA, K. D.; WILKINS, M.; ATIYEH, H. K.; DUNFORD, N. T.; HIZIROGLU, S. Effect of high dry solids loading on enzymatic hydrolysis of acid bisulfate pretreated Eastern redcedar. Bioresource Technol, v. 147, p. 168-176, 2013.

SANTOS, J. R. A.; GOUVEIA, E. R. Produção de bioetanol de bagaço de cana-de-açúcar. Rev Bras de Produtos Agroindustriais, v. 11, n. 1, p. 27-33, 2009.

SANTOS, F. A.; QUEIRÓZ, J.H.; COLODETTE, J.L.; FERNANDES, S.A.; GUIMARÃES, V. M.; REZENDE, S.T. Potencial da palha de cana-de-açúcar para produção de etanol. Quím. Nova, v. 35, p. 1004-1010, 2012

SANTOS F. A.; QUEIROZ, J. H.; COLODETTE, J. L.; MANFREDI, M.; QUEIROZ, M. E. L. R.; CALDAS, C. S.; SOARES, E. F. Otimização do pré-tratamento hidrotérmico da palha de canade-açúcar visando à produção de etanol celulósico. Quim. Nova, v.37, 56-62, 2013.

SILVA, V.F.N. Estudos de pré-tratamento e sacarificação enzimática de resíduos agroindustriais como etapas no processo de obtenção de etanol celulósico. 2009. 116 f. (Dissertação), Escola de Engenharia de Lorena - USP, Lorena, 2009. 